\section{Acute endophthalmitis after cataract surgery: 164 consecutive cases treated at a referral center in South Korea}

\begin{abstract}
Purpose To identify prognostic factors in patients referred with endophthalmitis after cataract surgery, and to evaluate the efficacy of primary vitrectomy as an initial management.

Methods Over an eight-year study period, we retrospectively reviewed the medical records of 164 patients who were referred with endophthalmitis following cataract surgery. Treatment generally conformed to standard guidelines, although primary vitrectomy was performed in several eyes with a visual acuity of hand motion or better, depending on the patient's status. Using multivariate analysis, we analyzed outcomes to determine the effect on final visual outcome.
\end{abstract}

Results A final visual acuity of $\geq 20 / 40$ was achieved in $92 / 164(56.1 \%)$ cases after

Department of

Ophthalmology, Kim's Eye Hospital, Myung-Gok Eye Research Institute, Konyang University College of Medicine, Seoul,

South Korea

\section{Correspondence:}

HJ Cho, Department of Ophthalmology, Kim's Eye Hospital, Konyang University College of medicine, 156, 4ga, Yeoungdeungpo-dong, Yeoungdeungpo-gu, Seoul 138-240, South Korea Tel: +82 22639 7777; Fax: +82 226397766. E-mail: chojoo@kimeye.com

Received: 17 November 2016

Accepted in revised form: 26 March 2017 Published online: 26 May 2017 treatment. Bacterial cultures showed bacterial growth in 89/164 cases (54.3\%). Among the various baseline characteristics, old age $(P=0.028)$, poor visual acuity at presentation $(P=0.004)$, gram-negative bacterial infection $(P=0.030)$, and short time between cataract surgery and signs of endophthalmitis $(P=0.021)$ were associated with poor visual outcome. The visual outcome showed no significant difference, in terms of initial treatment feature, between the primary vitrectomy with intraocular antibiotics injection (IOAI) and IOAI-only groups. However, reintervention was significantly less frequent in the primary vitrectomy group than in the IOAI group (12.5 and $32.7 \%$, respectively; $P=0.002$ ).

Conclusion Old age, poor visual acuity at presentation, type of cultured organism (gram-negative bacteria), and early onset of endophthalmitis after cataract surgery were significantly related to poor visual outcome after endophthalmitis treatment. Primary
SH Jeong, HJ Cho, HS Kim, Jl Han, DW Lee, CG Kim and JW Kim

vitrectomy may decrease the need for reintervention to control infection, although the treatment showed no benefits with regard to visual outcome.

Eye (2017) 31, 1456-1462; doi:10.1038/eye.2017.85; published online 26 May 2017

\section{Introduction}

Cataract surgery is one of the most commonly performed surgical procedures worldwide, and post-operative endophthalmitis is its most devastating complication. The incidence of endophthalmitis after cataract surgery by modern phacoemulsification has been reported as $0.02-0.08 \%$. $^{1,2}$

In cases of endophthalmitis, it is crucial that clinicians identify the causative organism and treat the patient using effective antibiotics. In addition, to determine as early as possible whether a patient requires aggressive therapy, physicians should evaluate a number of prognostic factors. Till date, initial visual acuity, ${ }^{3}$ history of diabetes mellitus or glaucoma, and the bacterial species involved have been reported as prognostic factors. ${ }^{4,5}$

The Endophthalmitis Vitrectomy Study (EVS) reported that immediate vitrectomy to treat endophthalmitis only benefits patients who present with a visual acuity of light perception (LP) or worse. ${ }^{3}$ However, more recently, vitrectomy technology has improved, and the efficacy of early vitrectomy for postoperative endophthalmitis also has also been reported. ${ }^{6}$ Currently, there is no consensus as to whether early vitrectomy is effective as an initial treatment for endophthalmitis after cataract surgery.

In the present study, we aimed to identify prognostic factors in patients referred with postoperative endophthalmitis after cataract surgery, and to analyze the various causative organisms 
in terms of clinical characteristics and visual outcome. We also evaluated-in the patients of a referral center-the therapeutic efficacy of primary vitrectomy as an initial treatment for endophthalmitis over a period of 8 years.

\section{Materials and methods}

To find patients who were referred from other local hospitals due to acute endophthalmitis after cataract surgery and were treated at Kim's eye hospital between January 2008 and December 2015, we performed a computerized search and medical record review. We excluded patients who had undergone combined surgery for vitreoretinal disease or glaucoma, as well as those who had a history of other intraocular surgery or penetrating trauma. The study was approved by the Institutional Review Board of Kim's Eye Hospital, Konyang University College of Medicine and conformed to the tenets of the Declaration of Helsinki (IRB No. A-2016-025).

Post-operative endophthalmitis was diagnosed when decreased visual acuity was combined with the following typical clinical features: marked intraocular inflammation, conjunctival injection, and hypopyon. Patients were referred from all over South Korea and were treated at all times of the day according to necessity. To manage postoperative endophthalmitis, clinicians generally followed the guidelines of the EVS, ${ }^{3}$ but not under a strict protocol. At baseline, every patient received an intraocular antibiotics injection (IOAI) of vancomycin hydrochloride $(1.0 \mathrm{mg}$ in $0.1 \mathrm{ml})$ and ceftazidime $(2.25 \mathrm{mg}$ in $0.1 \mathrm{ml})$. All injections were performed on the day of the diagnosis, without delay, and were administered in a clean surgical room. Primary vitrectomy was performed on every eye that presented with a visual acuity of LP. In patients with a visual acuity of more than hand motion (HM), primary vitrectomy was performed when (1) the cornea was severely edematous, or (2) there was loss of red reflex or severe vitreous opacity, regardless of visual acuity at presentation. Every pars plana vitrectomy was performed using $23 \mathrm{G}$ or $25 \mathrm{G}$ transconjunctival vitrectomy system. When inflammation did not improve within $48 \mathrm{~h}$ of initial treatment, an additional IOAI or vitrectomy was performed as a reintervention. Endophthalmitis was determined to have improved when visual acuity had increased, the vitreous media or anterior chamber had cleared, and pain had resolved.

At baseline, anterior chamber fluids and vitreous specimens were obtained, and an antibiotic sensitivity test was performed in every patient. During each vitrectomy, after all sclerotomies had been made, but before the fluid infusion was initiated, an initial undiluted vitreous specimen was obtained. Specifically, a vitreous cutter was inserted into the mid-vitreous, and $0.2-0.5 \mathrm{ml}$ of vitreous gel was manually aspirated into a syringe. Cultures from the samples were incubated on blood agar, chocolate agar, thioglycolate broth, and fresh Sabouraud dextrose agar. An antibiotic sensitivity test was carried out using the following antibiotics: ampicillin, ciprofloxacin, levofloxacin, vancomycin, erythromycin, gentamicin, and penicillin for Gram-positive organisms, and ciprofloxacin, levofloxacin, gentamicin, vancomycin, tetracycline, amikacin, and ceftazidime for gram-negative organisms. Antibiotic therapy initially consisted of broad-spectrum topical, intravitreal, and systemic antibiotics; this was subsequently modified in accordance with the results of the culture, sensitivity test, and clinical presentations. None of the patients were administered systemic steroids.

The following data were obtained from the medical records: sex, age, laterality of the eye involved, best correct visual acuity at each follow-up point, time from cataract surgery to symptoms of endophthalmitis, time from onset of endophthalmitis to referral, initial management at the local hospital before referral, treatment at the referral center, the presence of hypopyon at baseline, and results of bacterial cultures. We compared visual outcomes in patients who had undergone primary vitrectomy with those in patients who had received initial IOAI only; we also analyzed the outcomes of endophthalmitis in terms of which microbe was involved.

We analyzed continuous variables using a $t$-test, and categorical variables using a chi-square or Fisher's exact test. To investigate the relationship between baseline clinical characteristics and good visual outcome (more than 20/40 visual acuity at last visit), we used stepwise logistic regression. Specifically, we performed forward and backward stepwise regression using the likelihoodratio model, in which variables are selected using the likelihood-ratio statistic, which is based on maximum partial-likelihood estimates for each covariate. All statistical analyses were performed using the commercially available software package SPSS ver. 22.0 for Windows (SPSS Sciences, Chicago, IL, USA), and a $P$-value $<0.05$ was considered statistically significant.

\section{Results}

\section{Baseline characteristics}

A total of 164 eyes of 164 patients were included in the analysis. The mean age of the patients was 70.7 years (range: 41-96 years). The mean length of follow-up was 11.2 months (range: 3 months to 8 years, median:

6 months). The mean time from cataract surgery to the onset of endophthalmitis symptoms was 5.7 days (range: 1-30 days). In 81 eyes (49.4\%) endophthalmitis manifested within 3 days of surgery; in 47 eyes (28.6\%), it presented within 4-7 days of surgery; and in the 
remaining 36 eyes $(22.0 \%)$, it presented $>7$ days after surgery.

Patients were referred from all over South Korea, and most were referred immediately after developing signs of endophthalmitis. The median time from the development of endophthalmitis signs to referral was 1.8 days (range: 0-12 days). A total of 95 patients were referred within 3 days, 55 were referred within 4-7 days, and 14 were referred $>7$ days after developing signs of endophthalmitis.

Visual acuity at presentation was generally poor: in 140 patients $(85.4 \%)$ it was $\leq 20 / 400$. Furthermore, 86 patients $(52.4 \%)$ had a visual acuity of HM; in 25 patients $(15.2 \%)$ it was LP or less. The baseline characteristics of the patients are summarized in Table 1.

Most patients were referred without any intervention after the diagnosis of post-operative endophthalmitis. However, 34 of the 164 patients had undergone immediate treatment at their local hospital before referral: IOAI had been performed in 32 patients, and two patients had undergone vitrectomy before referral.

\section{Microbiological evaluation}

Of the 164 bacterial cultures, 89 (54.3\%) showed bacterial growth, and 75 (33.6\%) were negative for bacterial growth. Among the 89 positive cultures, 62 (69.7\%) were gram-positive, 23 (25.8\%) were gram-negative, and four $(4.5 \%)$ showed fungal growth. The most commonly isolated organism among the Gram-positive bacteria was Staphylococcus epidermidis (33 eyes); the second most common gram-positive bacterial species was Enterococcus faecalis (11 eyes). Among the gram-negative bacteria, Pseudomonas aeruginosa was the most frequently isolated (14 eyes). All Gram-positive isolates were susceptible to vancomycin; of the gram-negative isolates, $95 \%$ were susceptible to ceftazidime, and $80.0 \%$ were susceptible to levofloxacin.

Gram-positive bacteria showed significantly higher rates of good visual outcome (visual acuity was more than 20/40) than gram-negative bacteria (56.5 and 34.8\%, respectively; $P=0.026$ ). Among the causative organisms that occurred in more than five patients, the rate of good visual outcome was highest in cases of Staphylococcus epidermidis $(69.7 \%)$, and lowest in cases of Pseudomonas aeruginosa $(21.4 \%)$. The results of the bacterial cultures, with corresponding final visual acuities, are shown in Table 2.

\section{Treatment features and final visual acuity}

Primary vitrectomy with IOAI was performed in 112 of 164 cases $(68.3 \%)$, and only IOAI was performed in 52 of 164 cases $(31.7 \%)$. All cases of initial visual acuity less
Table 1 Baseline characteristics of patients referred due to endophthalmitis after cataract surgery

\begin{tabular}{|c|c|}
\hline & Total $(\mathrm{n}=164)$ \\
\hline Age & $\begin{array}{c}70.7 \pm 8.9 \text { (range: } \\
41-96)\end{array}$ \\
\hline Mean follow-up period (months) & $\begin{array}{c}11.2 \pm 12.8 \text { (range: } \\
3-96)\end{array}$ \\
\hline \multicolumn{2}{|l|}{ Sex, n $(\%)$} \\
\hline Men & $81(49.4)$ \\
\hline Women & $83(50.6)$ \\
\hline \multicolumn{2}{|l|}{ Involved eye, $\mathrm{n}(\%)$} \\
\hline Right & $80(48.8)$ \\
\hline Left & $84(51.2)$ \\
\hline \multicolumn{2}{|l|}{ Presenting visual acuity, n (\%) } \\
\hline LP or less & $25(15.2)$ \\
\hline $\mathrm{HM}$ & $86(52.4)$ \\
\hline CF or better & $53(32.3)$ \\
\hline \multicolumn{2}{|l|}{ Time from surgery to presentation, $\mathrm{n}(\%)$} \\
\hline $1-3$ days & $81(49.4)$ \\
\hline $4-7$ days & $47(28.7)$ \\
\hline$>7$ days & $36(22.0)$ \\
\hline \multicolumn{2}{|l|}{ Type of cataract surgery, $\mathrm{n}(\%)$} \\
\hline Phacoemulsification & $158(96.3)$ \\
\hline ECCE & $6(3.7)$ \\
\hline $\begin{array}{l}\text { Presence of hypopyon at presentation, } \\
n(\%)\end{array}$ & $134(81.7)$ \\
\hline $\begin{array}{l}\text { Ruptured posterior capsule at } \\
\text { presentation, } n(\%)\end{array}$ & $7(4.3)$ \\
\hline $\begin{array}{l}\text { Patients who received treatment at local } \\
\text { hospitals before referral, } n(\%)\end{array}$ & $39(23.8)$ \\
\hline Intraocular pressure at baseline & $\begin{array}{c}14.5 \pm 6.5 \text { (range: } \\
6-37 \text { ) }\end{array}$ \\
\hline
\end{tabular}

Abbreviations: $\mathrm{CF}$, counting finger; ECCE, extracapsular cataract extraction; HM, hand motion; LP, light perception.

than LP were treated with primary vitrectomy (25 eyes; $15.2 \%$ ). When initial visual acuity was HM, primary vitrectomy was performed in 60 out of 86 cases, and only IOAI was performed in 26 of 86 cases.

Good visual outcome (final visual acuity $>20 / 40$ ) was achieved in 58 of the 112 eyes (51.8\%) that had undergone primary vitrectomy and IOAI, and in 34 of the 52 eyes $(65.4 \%)$ that had undergone IOAI only; this was not a significant difference $(P=0.102)$. In a subgroup analysis of patients with a baseline visual acuity more than HM, the proportion of patients with good visual outcome did not differ significantly between the primary vitrectomy and IOAI groups (Table 3).

However, reintervention after the initial treatment was required significantly more frequently in the IOAI group than in the vitrectomy group $(P=0.002$; Table 4$)$. Moreover, 14 of the 112 eyes $(12.5 \%)$ that had initially been treated using primary vitrectomy and IOAI 
Table 2 Results of bacterial cultures in patients referred due to endophthalmitis after cataract surgery

\begin{tabular}{|c|c|c|}
\hline & Total $(\mathrm{n}=89)$ & $\begin{array}{c}\text { Good visual outcome } \\
\text { (more than 20/40 at } \\
\text { final visit) }\end{array}$ \\
\hline Gram-positive bacteria & $62(69.7 \%)$ & $35(56.5 \%)$ \\
\hline $\begin{array}{l}\text { Staphylococcus } \\
\text { epidermidis }\end{array}$ & $33(37.1 \%)$ & $23(69.7 \%)$ \\
\hline$M R S A$ & $1(1.1 \%)$ & $1(100 \%)$ \\
\hline Streptococcus sanguinis & $4(4.5 \%)$ & $1(25 \%)$ \\
\hline Streptococcus mitis & $4(4.5 \%)$ & $2(50 \%)$ \\
\hline Streptococcus pneumoniae & $3(3.4 \%)$ & $1(33.3 \%)$ \\
\hline Streptococcus agalactiae & $2(2.2 \%)$ & $2(100 \%)$ \\
\hline Staphylococcus & $2(2.2 \%)$ & $1(50 \%)$ \\
\hline \multicolumn{3}{|l|}{ lugdunensis } \\
\hline Streptococcus salivarius & $2(2.2 \%)$ & $1(50 \%)$ \\
\hline Enterococcus faecalis & $11(12.4 \%)$ & $5(45.5 \%)$ \\
\hline Gram-negative bacteria & $23(25.8 \%)$ & $8(34.8 \%)$ \\
\hline Pseudomonas aeruginosa & $14(15.7 \%)$ & $3(21.4 \%)$ \\
\hline Acinetobacter baumannii & $2(2.2 \%)$ & $2(100 \%)$ \\
\hline Serratia marcescens & $1(1.1 \%)$ & $0(0 \%)$ \\
\hline Klebsiella pneumoniae & $1(1.1 \%)$ & $1(100 \%)$ \\
\hline $\begin{array}{l}\text { Alcaligenes xylosoxidans } \\
\text { ss. denitrificans }\end{array}$ & $1(1.1 \%)$ & $1(100 \%)$ \\
\hline $\begin{array}{l}\text { Stenotrophomonas } \\
\text { maltophilia }\end{array}$ & $4(4.5 \%)$ & $1(25 \%)$ \\
\hline Fungus & $4(4.5 \%)$ & $1(25 \%)$ \\
\hline Aspergillus sp. & $2(2.2 \%)$ & $0(0 \%)$ \\
\hline Candida sp. & $2(2.2 \%)$ & $1(50 \%)$ \\
\hline
\end{tabular}

Abbreviation: MRSA, Methicillin-resistant Staphylococcus epidermidis.

Table 3 Proportions of good visual outcome (more than 20/40 visual acuity) after endophthalmitis treatment: a comparison between primary vitrectomy and intravitreal antibiotic injection as initial treatments

\begin{tabular}{lrrrr}
\hline & \multicolumn{3}{c}{ Final $V A \geq 20 / 40$} & \multirow{2}{*}{$P^{\mathrm{a}}$} \\
\cline { 2 - 3 } & & $n$ & $\%$ & \\
\hline All cases & 164 & & & \\
$\quad$ Primary vitrectomy with IOAI & 112 & 58 & 51.8 & 0.128 \\
IOAI only & 52 & 34 & 65.4 & \\
Cases with presenting VA of HM & 86 & & & \\
$\quad$ Primary vitrectomy with IOAI & 60 & 34 & 56.7 & 0.809 \\
IOAI only & 26 & 14 & 53.8 & \\
Cases with presenting VA more & 139 & & & \\
than HM & & & & \\
$\quad$ Primary vitrectomy with IOAI & 87 & 54 & 62.1 & 0.695 \\
IOAI only & 52 & 34 & 65.4 & \\
\hline
\end{tabular}

Abbreviations: $\mathrm{CF}$, counting finger; $\mathrm{HM}$, hand motion; IOAI, intraocular antibiotics injection; LP, light perception; VA, visual acuity. ${ }^{\mathrm{a} C h i-}$ Square test.

underwent an additional vitrectomy or IOAI, whereas 17 out of the 52 cases $(32.7 \%)$ that had initially been treated using only IOAI underwent an additional vitrectomy or IOAI to control inflammation. A subgroup analysis of patients with a visual acuity of more than HM at
Table 4 Patients with endophthalmitis who required reintervention after initial treatment

\begin{tabular}{|c|c|c|c|c|}
\hline \multirow[t]{2}{*}{ Initial treatment } & & \multicolumn{2}{|c|}{$\begin{array}{l}\text { Patients } \\
\text { requiring } \\
\text { reintervention }\end{array}$} & \multirow[t]{2}{*}{$P^{\mathrm{a}}$} \\
\hline & & $n$ & $\%$ & \\
\hline All cases & 164 & & & 0.002 \\
\hline Primary vitrectomy with IOAI & 112 & 14 & 12.5 & \\
\hline IOAI only & 52 & 17 & 32.7 & \\
\hline $\begin{array}{l}\text { Cases with presenting visual } \\
\text { acuity } \geq \mathrm{HM}\end{array}$ & 139 & & & 0.001 \\
\hline Primary vitrectomy with IOAI & 87 & 9 & 10.3 & \\
\hline IOAI only & 52 & 17 & 32.7 & \\
\hline
\end{tabular}

Abbreviations: $\mathrm{CF}$, counting finger; $\mathrm{HM}$, hand motion; IOAI, intraocular antibiotics injection; LP, light perception; VA, visual acuity. ${ }^{a}$ ChiSquare test.

presentation revealed that among the 52 eyes that had initially been treated using IOAI only, 17 (32.7\%)

eventually required reintervention with vitrectomy. This was significantly higher than the reintervention requirement in the primary vitrectomy group (10.3\%; $P=0.001$; Table 4).

\section{Good visual outcome and associated factors}

Overall, $56.1 \%$ of the patients (92 out of 164) showed good visual outcome after treatment. Among the baseline characteristics, age, sex, visual acuity at presentation, time from cataract surgery to signs of endophthalmitis, presence of hypopyon at presentation, positivity of microbial culture, causative organism, pre-treatment before referral, and primary vitrectomy as an initial treatment were identified as possible predictive factors of visual outcome.

Stepwise regression analysis showed that four of these factors were significantly associated with poor visual outcome: old age (odds ratio (OR): 1.240; 95\% confidence interval (CI): 1.112-1.526; $P=0.028$ ), poor presenting visual acuity (OR: 1.114; 95\% CI: 1.090-1.336; $P=0.004$ ), gram-negative bacterial infection (OR: 1.031; 95\% CI: 1.019-1.268; $P=0.030)$, and short time between cataract surgery and appearance of signs of endophthalmitis (OR: 2.026; 95\% CI: 1.075-3.114; $P=0.021$; Table 5).

\section{Discussion}

Although advancements have been made in terms of prophylaxis and surgical techniques, endophthalmitis remains the most serious complication of cataract surgery. Previous studies have reported that the proportion of patients with good visual acuity $(>20 / 40)$ after endophthalmitis treatment is $28-53 \% .^{3,7-10}$ In the current study, good visual outcome was achieved in $56.7 \%$ of the 
Table 5 Logistic regression analysis using a dependent variable of good visual outcome (more than 20/40 visual acuity) after treatment for endophthalmitis secondary to cataract surgery

\begin{tabular}{|c|c|c|c|c|}
\hline \multirow[t]{2}{*}{ Variable } & \multicolumn{2}{|c|}{ Univariate analysis } & \multicolumn{2}{|c|}{ Multivariate analysis } \\
\hline & OR $(95 \% \mathrm{CI})$ & $P$ & OR $(95 \% \mathrm{CI})$ & $P$ \\
\hline Age & $1.256(1.109-1.743)$ & 0.019 & $1.240(1.112-1.526)$ & 0.028 \\
\hline Gender & $1.052(1.011-1.418)$ & 0.038 & $1.032(0.995-1.171)$ & 0.069 \\
\hline Presenting visual acuity (logMAR) & $1.128(1.050-1.417)$ & 0.001 & $1.114(1.090-1.336)$ & 0.004 \\
\hline $\begin{array}{l}\text { Time from cataract surgery to signs of endophthalmitis } \\
\text { ( }<3 \text { days or } \geq 3 \text { days) }\end{array}$ & $2.648(1.051-4.126)$ & 0.011 & $2.026(1.075-3.114)$ & 0.021 \\
\hline Hypopyon at presentation (yes or no) & $3.185(0.929-6.672)$ & 0.119 & & \\
\hline Positive result of microbial culture (yes or no) & $1.009(0.912-1.266)$ & 0.662 & & \\
\hline Causative organism (Gram-positive or negative) & $1.145(1.010-1.418)$ & 0.021 & $1.031(1.019-1.268)$ & 0.030 \\
\hline Pre-treatment before referral (yes or no) & $0.946(0.942-1.442)$ & 0.451 & & \\
\hline Primary vitrectomy as initial treatment (yes or no) & $0.823(0.427-1.362)$ & 0.139 & & \\
\hline
\end{tabular}

Abbreviations: CI, confidence interval; logMAR, logarithm of minimal angle of resolution; OR, odd ratio.

patients, which is consistent with the results of the EVS study (53\%). ${ }^{3}$ The treatment outcomes of endophthalmitis have not changed much since the EVS of the mid-nineties.

It has been reported that the predictive factors of postendophthalmitis visual prognosis include the following: the causative organism, visual acuity at presentation, and treatment type. ${ }^{3,11}$ In the current study, poor visual outcome after treatment was associated with old age, poor visual acuity at presentation, infection by a gramnegative microorganism, and short time from cataract surgery to signs of endophthalmitis.

Previous studies have reported an association between the causative organism and prognosis. Specifically, the EVS demonstrated an inverse relationship between better final visual outcome and the likelihood of Gram-negative infection. ${ }^{3}$ In the current study, 56.5\% patients with a Gram-positive bacterial infection (35 out of 62 eyes) achieved good visual acuity after treatment. Conversely, 8 out of 23 eyes $(34.8 \%)$ with a gram-negative bacterial infection achieved $>20 / 40$ visual acuity; this result is similar to that of previous reports $(28.5 \%$ and $38.9 \%$, respectively). ${ }^{5,12}$ In particular, Pseudomonas aeruginosa infection, which was the most frequent gram-negative infection in the present study, showed the lowest rate of good visual outcome, achieving 20/40 visual acuity in only $21.4 \%$ of cases. Indeed, several investigations have reported poor visual outcomes for Pseudomonas aeruginosa endophthalmitis, even when the organism is susceptible to the antibiotics administered, and with early and appropriate treatment. ${ }^{13}$ The detection of gram-negative species in early stage of endophthalmitis could be crucial for proper management.

Besides causative organisms, a short time from cataract surgery to signs of endophthalmitis was strongly associated with treatment outcome in the current study. Among the patients with Pseudomonas-associated endophthalmitis, most (10 out of 14 eyes; $71.4 \%$ ) had shown signs of endophthalmitis within 3 days of cataract surgery. On the other hand, most cases of Staphylococcus epidermidis-associated endophthalmitis (24 out of 33 eyes; $72.7 \%$ ), which showed the highest proportion of good visual outcome, developed signs of endophthalmitis signs more than 3 days after surgery. Not only may fast onset and progression of inflammation reflect the host's response to infection, they may also indicate high virulence in the causative bacteria, with a correspondingly worse expected treatment outcome. ${ }^{14,15}$ Therefore, clinicians should be aware that, when endophthalmitis develops in the early post-operative phase after cataract surgery and progression is rapid, it is likely that the endophthalmitis involves a high-virulence microorganism infection, and that the risk of poor visual outcome is higher.

Endophthalmitis management has not changed considerably since the EVS report of 1995. The EVS only advocated primary vitrectomy in patients with a visual acuity of LP or worse at presentation. The same study included the patients with clear cornea enough to allow vitrectomy and exclusion of patients who did not consent to therapeutic randomization. ${ }^{3}$ However, current vitrectomy machines offer a sutureless, small-gauge system, increased safety close to the retina, and increased visibility throughout the entire surgical field via panoramic viewing. ${ }^{6}$ Moreover, vitrectomy may directly remove the infectious and inflammatory load from the eye. This raises the question of whether vitrectomy should indeed only be performed in patients with a visual acuity of LP, and of whether physicians should wait until the visual acuity has deteriorated to LP before performing the procedure. There is no current consensus about the therapeutic efficacy of primary vitrectomy for endophthalmitis.

In the current study, good visual outcome was not significantly different between the primary vitrectomy 
and IOAI only groups. In a subgroup of patients who presented with a visual acuity more than HM, which denotes better visual acuity than LP, no significant difference was found between the initial treatments in terms of the proportion of patients who achieved good visual acuity (54 out of $87(62.1 \%)$ eyes that had undergone primary vitrectomy; 34 out of 52 eyes (65.4\%) that had undergone IOAI only). However, reintervention was required more frequently in the IOAI-only group than in the primary vitrectomy group. Among 52 patients who had presented with a visual acuity of more than HM and had undergone IOAI only as an initial treatment, $17(32.7 \%)$ eventually required vitrectomy to control inflammation. Our results suggest that primary vitrectomy decreases the need for repeated interventions and shortens the treatment period or recovery time of endophthalmitis, although it may not affect final visual outcome. Additionally, when vitrectomy is delayed on the basis of visual acuity only, inflammation on the cornea may result in poor visualization during surgery; that is, a poor pre-operative ocular condition can lead to a higher complication rate. Therefore, vitrectomy may be an effective initial treatment, even when the presenting visual acuity is more than HM, which is better than a visual acuity of LP. The definitive indications or therapeutic efficacy of immediate vitrectomy for endophthalmitis should be investigated more thoroughly with prospective studies.

The current study had several limitations, besides its retrospective nature. First, vitrectomy was selected as an initial treatment or reintervention according to each physician's discretion without referring to any established criteria. In addition, we could not evaluate the potential impact of factors such as intracameral antibiotics, localization, construction of corneal incisions, or preoperative preparation such as povidone-iodine. Another limitation was that we could not evaluate the efficacy of adding steroids to the IOAI; steroid injection is not generally performed in our institution. There is a still controversy in this regard, and the results of previous investigations vary; some reports indicate no effect while others have indicated a beneficial effect on treatment outcome. ${ }^{16,17}$ In fact, treatment outcomes in the present study were consistent with those of the EVS study, even though we used no steroid adjuvant. The effects of adding steroids to endophthalmitis treatment regimen should be investigated with prospective studies in the near future. Furthermore, there was the possibility of treatment delay, as all patients in the study were referred cases. The median time from the development of endophthalmitis signs to referral was 1.8 days. However, immediate appropriate management was provided for the patients at the institution, and the treatment results of this study were similar to those of previous reports.
In summary, old age, poor visual acuity at presentation, type of cultured organism (gram-negative bacteria), and early onset of endophthalmitis after cataract surgery are significantly related to poor visual outcome after endophthalmitis treatment. When a poor visual outcome is expected at baseline, more aggressive treatment should be performed. Primary vitrectomy did not prove to be more favorable than IOAI in terms of visual outcome; however, immediate vitrectomy could decrease requirement for reintervention for achieving infection control. Further studies should be conducted in near future to investigate the role of primary vitrectomy in the management of acute endophthalmitis.

\section{Summary}

What was known before

- There is no current consensus about the therapeutic efficacy of primary vitrectomy for endophthalmitis for patients with a visual acuity of hand motion or better

What this study adds

- Immediate vitrectomy for acute postoperative endophthalmitis with good visual acuity could decrease requirement for reintervention for achieving infection control

\section{Conflict of interest}

The authors declare no conflict of interest.

\section{References}

1 Endophthalmitis Study Group, European Society of Cataract \& Refractive Surgeons. Prophylaxis of postoperative endophthalmitis following cataract surgery: results of the ESCRS multicenter study and identification of risk factors. J Cataract Refract Surg 2007; 33(6): 978-988.

2 Kattan HM, Flynn Jr HW, Pflugfelder SC, Robertson C, Forster RK. Nosocomial endophthalmitis survey. Current incidence of infection after intraocular surgery. Ophthalmology 1991; 98(2): 227-238.

3 Results of the Endophthalmitis Vitrectomy Study. A randomized trial of immediate vitrectomy and of intravenous antibiotics for the treatment of postoperative bacterial endophthalmitis. Endophthalmitis Vitrectomy Study Group. Arch Ophthalmol 1995; 113(12): 1479-1496.

$4 \mathrm{Ng}$ JQ, Morlet N, Pearman JW, Constable IJ, McAllister IL, Kennedy CJ et al. Management and outcomes of postoperative endophthalmitis since the endophthalmitis vitrectomy study: the Endophthalmitis Population Study of Western Australia (EPSWA)'s fifth report. Ophthalmology 2005; 112(7): 1199-1206.

5 Lalwani GA, Flynn HW, Scott IU, Quinn CM, Berrocal AM, Davis JL et al. Acute-onset endophthalmitis after clear corneal cataract surgery (1996-2005). Clinical features, 
causative organisms, and visual acuity outcomes. Ophthalmology 2008; 115(3): 473-476.

6 Kuhn F, Gini G. Ten years after... are findings of the Endophthalmitis Vitrectomy Study still relevant today? Graefe's Arch Clin Exp Ophthalmol 2005; 243(12): 1197-1199.

7 Kim HW, Kim SY, Chung IY, Lee JE, Lee JE, Park JM et al. Emergence of Enterococcus species in the infectious microorganisms cultured from patients with endophthalmitis in South Korea. Infection 2014; 42(1): 113-118.

8 Eifrig CW, Flynn Jr HW, Scott IU, Newton J. Acute-onset postoperative endophthalmitis: review of incidence and visual outcomes (1995-2001). Ophthalmic Surg Lasers 2002; 33(5): 373-378.

9 Lertsumitkul S, Myers PC, O'Rourke MT, Chandra J. Endophthalmitis in the western Sydney region: a casecontrol study. Clin Exp Ophthalmol 2001; 29(6): 400-405.

10 Friling E, Lundstrom M, Stenevi U, Montan P. Six-year incidence of endophthalmitis after cataract surgery: Swedish national study. J Cataract Refract Surg 2013; 39(1): 15-21.

11 Combey de Lambert A, Campolmi N, Cornut PL, Aptel F, Creuzot-Garcher C, Chiquet C et al. Baseline factors predictive of visual prognosis in acute postoperative bacterial endophthalmitis in patients undergoing cataract surgery. JAMA Ophthalmol 2013; 131(9): 1159-1166.
12 Endophthalmitis Vitrectomy Study Group. Microbiologic factors and visual outcome in the endophthalmitis vitrectomy study. Am J Ophthalmol 1996; 122(6): 830-846.

13 Sridhar J, Kuriyan AE, Flynn HW, Miller D. Endophthalmitis caused by Pseudomonas Aeruginosa: clinical features, antibiotic susceptibilities, and treatment outcomes. Retina 2015; 35(6): 1101-1106.

14 Kamalarajah S, Silvestri G, Sharma N, Khan A, Foot B, Ling $\mathrm{R}$ et al. Surveillance of endophthalmitis following cataract surgery in the UK. Eye 2004; 18(6): 580-587.

15 Johnson MW, Doft BH, Kelsey SF, Barza M, Wilson LA, Barr CC et al. The Endophthalmitis Vitrectomy Study. Relationship between clinical presentation and microbiologic spectrum. Ophthalmology 1997; 104(2): 261-272.

16 Gan IM, Ugahary LC, van Dissel JT, Feron E, Peperkamp E, Veckeneer $\mathrm{M}$ et al. Intravitreal dexamethasone as adjuvant in the treatment of postoperative endophthalmitis: a prospective randomized trial. Graefes Arch Clin Exp Ophthalmol 2005; 243(12): 1200-1205.

17 Shah GK, Stein JD, Sharma S, Sivalingam A, Benson WE, Regillo CD et al. Visual outcomes following the use of intravitreal steroids in the treatment of postoperative endophthalmitis. Ophthalmology 2000; 107(3): 486-489. 